\title{
Wide field-of-view bifocal eyeglasses
}

\author{
Sergio Barbero ${ }^{a}$ and Jacob Rubinstein ${ }^{b}$ \\ ${ }^{a}$ Instituto de Óptica (CSIC), Serrano 121, 28006, Madrid, Spain. \\ ${ }^{b}$ Department of Mathematics, Technion, Haifa 32000, Israel.
}

\begin{abstract}
When vision is affected simultaneously by presbyopia and myopia or hyperopia, a solution based on eyeglasses implies a surface with either segmented focal regions (e.g. bifocal lenses) or a progressive addition profile (PALs). However, both options have the drawback of reducing the field-of-view for each power position, which restricts the natural eye-head movements of the wearer. To avoid this serious limitation we propose a new solution which is essentially a bifocal power-adjustable optical design ensuring a wide field-of-view for every viewing distance. The optical system is based on the Alvarez principle. Spherical refraction correction is considered for different eccentric gaze directions covering a field-of-view range up to 45degrees. Eye movements during convergence for near objects are included. We designed three bifocal systems. The first one provides $3 \mathrm{D}$ for far vision (myopic eye) and $-1 \mathrm{D}$ for near vision (+2 D Addition). The second one provides a $+3 \mathrm{D}$ addition with $3 \mathrm{D}$ for far vision. Finally the last system is an example of reading glasses with $+1 \mathrm{D}$ power Addition.
\end{abstract}

Keywords: bifocal spectacles, power adjustable lenses, Alvarez lenses, wide field-of-view optics

\section{INTRODUCTION}

Presbyopia (age-related diminished ability to focus on near objects) affects a large part of the population: most people above the age of 45 . Besides, in many cases presbyopia is accompanied by myopia or hyperopia, that is a loss of focus for far vision. In the presence of both defects the image is differently blurred when looking a far with respect to a near object. Consequently monofocal spectacles are not a satisfactory solution. The typical alternative, still based on eyeglasses, implies the use of segmented focal regions (bifocal or trifocal lenses) or progressive addition surfaces (PALs).

In bifocal lenses the smaller part, located nearer to the nasal region, is designed for near-distance vision (thus providing higher optical power) whereas the larger part is used for far-distance vision. The jump (abrupt discontinuity) between the distance and the near regions of the lens generates discontinuities in the prismatic effects that can lead to undesired visual effects. ${ }^{1}$ The importance of prismatic errors in bifocal designs was early contemplated. ${ }^{2}$

As an alternative PALs designs appeared at the mid-twentieth century. Here the lens surface is smooth, and the optical power changes gradually as the eye moves downwards and in the nasal direction, thus providing a continuous power change. However, PALs have the drawback of reducing the field-of-view for each power position (far, near or intermediate object locations), which restricts the natural eye-head movements of the wearer.

To avoid these serious limitations of traditional bifocals and PALs we propose a novel type of bifocal power-adjustable lenses which ensure a wide field-of-view for every viewing distance without the problem of discontinuities. For this work we limited ourselves to two (bifocal) object locations, although the design could be extended to include other vision conditions (e.g. three object locations, i.e. a trifocal design), obviously sacrificing some field-of-view.

Following this philosophy a solution based on a deformable lens structure was proposed by Barnea ${ }^{3}$ (original trade name Trufocals, currently Superfocus). A fluid is confined by two transparent membranes and the variable power is obtained by imposing forces on the periphery of the membranes which varies the volume of the fluid in a manner to induce a desired power change. However here we follow a different approach based on Alvarez's

Further author information, E-mail: sergio.barbero@csic.es

Optical Systems Design 2015: Optical Design and Engineering VI, edited by Laurent Mazuray,

Rolf Wartmann, Andrew P. Wood, Proc. of SPIE Vol. 9626, 962614 - (c) 2015

SPIE $\cdot$ CCC code: $0277-786$ X/15/\$18 $\cdot$ doi: $10.1117 / 12.2184596$

Proc. of SPIE Vol. 9626 962614-1 
principle, which from our point of view ensures a better optical performance. This principle is based on the fact that two lenses with cubic-type surfaces laterally shifted one with respect to the other can induce a spherical power change. In ophthalmic optics Alvarez lenses has been revived in recent years with the purpose of supplying affordable adjustable lenses to solve the functional blindness problem in developing countries due to uncorrected refractive errors. ${ }^{4-8}$

\section{DESIGN METHODOLOGY}

An Alvarez composite optical system comprises two lenses for each eye. The lenses have a planar and a cubictype surface which are arranged with their planar surfaces in contact, so that the incoming light is only refracted by two surfaces. The non-planar surfaces, which are the ones to be designed, can be third order polynomials described by nine terms. ${ }^{4,5}$

We have previously designed these types of lenses for spherical refraction correction ${ }^{4}$ and sphero-cylindrical refraction measurement. ${ }^{5}$ Other designs for adjustable spherical correction have been published. ${ }^{9}$ In all these designs the optical axis is kept fixed between different focal positions. However when the spectacles are used to correct vision for different viewing distances the designer must take into account that the principal gaze direction changes for each object location and consequently the optical axis is displaced.

Figure 1 illustrate this effect. Typically near viewing objects are located downwards (e.g. a book) so the eye rotates vertically $\left(\alpha_{y}\right.$ in Fig. 1(a)). Simultaneously both eyes converge to look to a common point (point $P$ at Fig. 1(b)). This convergence forces an horizontal eye rotation $\alpha_{x}$ (Fig. 1(b)). Both rotations generate a new optical axis passing through a point in the spectacles located a distance $\left(L_{x}, L_{y}\right)$ from the principal viewing direction for far vision.

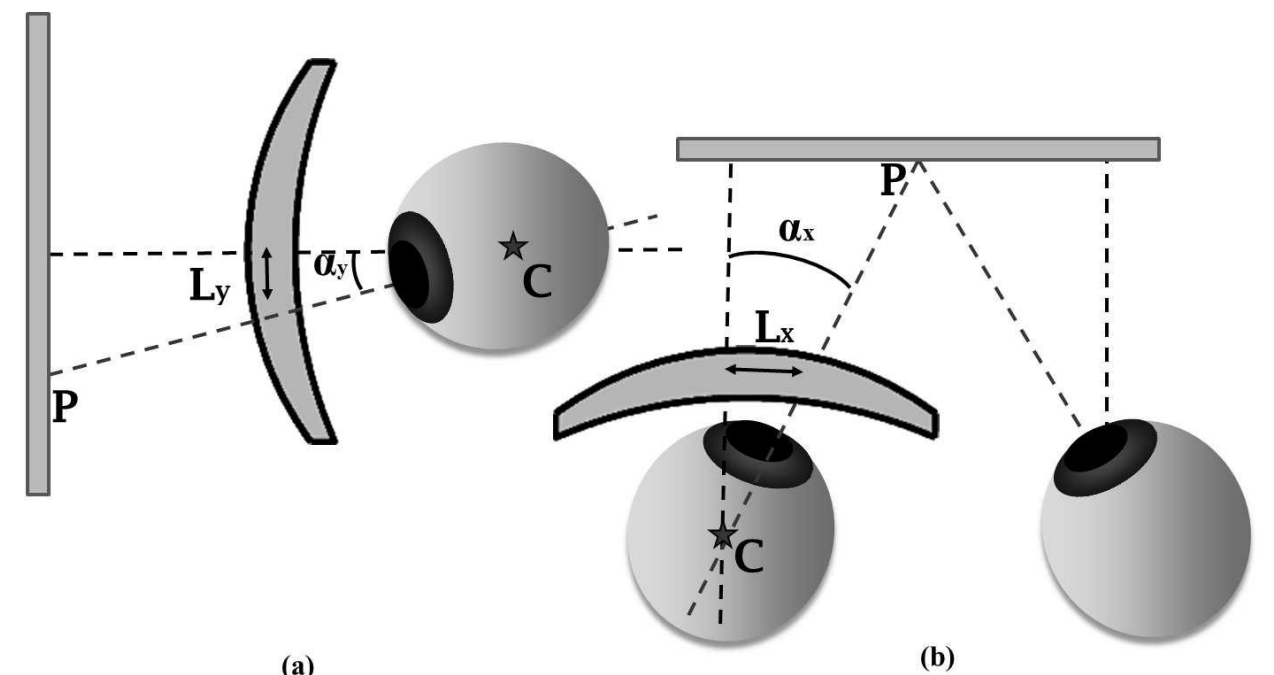

(a)

(b)

Figure 1. Eye movement when looking towards a near object point P: (a) Vertical rotation (b) Horizontal (towards nasal) rotation

Our design methodology is similar to the one published by us before ${ }^{4,5}$ but introducing some novelties to include the change in the optical axis for different viewing directions. In this paper we concentrate on a bifocal design, hence we used the standard notation and available data from typical bifocal eyeglasses. The distance visual point (DVP) and the near visual point (NVP) are defined as the points on the lens used for distance and near vision respectively when the eye rotates towards its primary gaze directions. In bifocal eyeglasses the NVP is located typically $2 \mathrm{~mm}$ inwards and $4 \mathrm{~mm}$ downwards from the DVP (p. $141^{1}$ ), although we note that these values could vary because of several factors. The reading addition, or just the Addition (Add), is the difference in vertex power when looking through the DVP with respect to NVP. 
The non-planar surfaces of our Alvarez designs take the form: ${ }^{4}$

$$
u(x, y)=\frac{c r^{2}}{1+\sqrt{1-(K+1) c r^{2}}}+p_{1} x^{3}+p_{2} y x^{2}+p_{3} x y^{2}+p_{4} y^{3}+p_{5} x y+p_{6} x+p_{7} y .
$$

Here $r=\sqrt{x^{2}+y^{2}}$, and $\mathrm{c}$ and $\mathrm{K}$ are the radius of curvature and the asphericity of the base conic.

The lateral shift must be applied to both lenses. However, whereas in previous designs the shift is the same for both lenses ${ }^{4,5}$ in the current one, this distance is, in general, different because the change in optical axis. Therefore a special mechanical design must be used. ${ }^{10}$ We note that the strong difficulties in designing a mechanical set-up with vertical movements recommend to opt for a design with only horizontal movements, ${ }^{10}$ leaving the vertical re-centering to be made manually. Hence from now on we only consider horizontal changes in the optical axis

The method to compute the actual power and astigmatism distribution across the lens is based on differential ray tracing. ${ }^{411-13}$ In this paper we have only included base surfaces with spherical refraction correction, although a base curve including sphero-cylindrical correction could be included, as proposed by Peloux et al. ${ }^{9}$

We constructed a merit function given by:

$E=\int\left(w_{1}(x, y)\left(S_{1}(x, y)-S\right)^{2}+v_{1}(x, y) C_{1}(x, y)^{2}\right)+\int\left(w_{2}(x, y)\left(S_{1}(x, y)-(S+A d d)\right)^{2}+v_{2}(x, y) C_{2}(x, y)^{2}\right)$

where $S_{1}(x, y)$ and $C_{1}(x, y)$ are the actual optical power and cylinder, respectively, of the optical composite lens when the eye gazes at far distance and $S_{2}(x, y)$ and $C_{2}(x, y)$ are the same magnitudes when the eye looks at a near distance object. The spherical component of the refraction prescription for far vision is given by $S$ (zero cylinder) and $A d d$ is the power addition. Finally $w_{1}, w_{2}, v_{1}$ and $v_{2}$ are arbitrary weights to be used. The design procedure consists of modifying the parameters of Eq. 1, through a cascade optimization, ${ }^{4}$ until Eq. 2 is minimized.

The optimization of Eq. 2 highly depends on the initial parameters describing the surfaces (parameters of Eq. 1), specially the $p_{1}$ term. In the appendix of this paper we provide a method to obtain a reasonable initial value for $p_{1}$.

\section{DESIGN EXAMPLES}

In our first example, our design aims at providing wide field-of-view performance for far-distance and neardistance viewing to a person who has a left-eye far-distance prescription given by $S)$, zero cylinder $(C=0)$ and an addition Add of $+2.0 \mathrm{D}$. The central thickness of the front lens is $1.4 \mathrm{~mm}$ and that of the back lens is 2.4 $\mathrm{mm}$. The refractive index of both lenses is $\mathrm{n}=1.586$.

For the "rest position" (no lens movement) the optical power at a point aligned with the primary gaze direction is $2.3 \mathrm{D}$. When the lenses are moved to a first relative position, achieved by a horizontal shift of 2 $\mathrm{mm}$ of the front lens in the negative $\mathrm{x}$-direction from the rest position, the optical power at a point aligned with the primary gaze direction is 3 diopters. While the lenses are in a second relative position, achieved by a horizontal shift of $4 \mathrm{~mm}$ of the front lens in the positive $\mathrm{x}$-direction from the rest position, the optical power is $1 \mathrm{D}$.

Fig. 2 shows the power (a-c) and cylinder (b-d) error distribution (deviations from nominal values) for far $(\mathrm{a}-\mathrm{b})$ and near $(\mathrm{c}-\mathrm{d})$ vision viewing conditions.

The graphic shows that the deviation, for both sphere and cylinder, is less than 0.25 diopters within an elliptical optical window having major and minor axes of at least 40 degrees by 40 degrees of eye rotation.

The second design example is again a bifocal design but with an addition of $+3 \mathrm{D}$. The higher addition imposes larger thicknesses: $2.25 \mathrm{~mm}$ for the front lens and $3 \mathrm{~mm}$ for the back lens. Fig. 3 shows the power (a-c) and cylinder (b-d) error distribution (deviations from nominal values) for far (a-b) and near (c-d) vision configuration. 


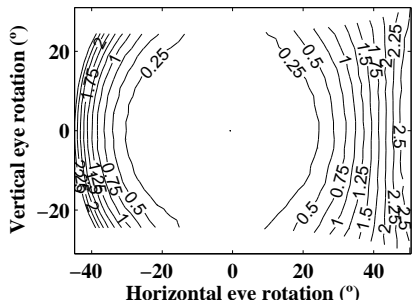

(a)

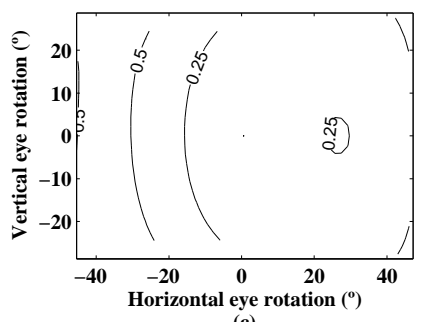

(c)

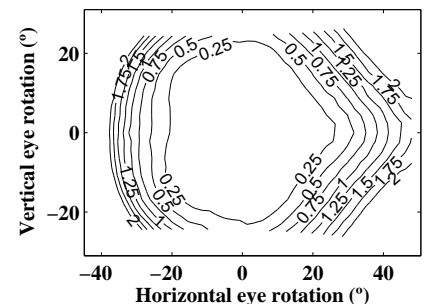

(b)

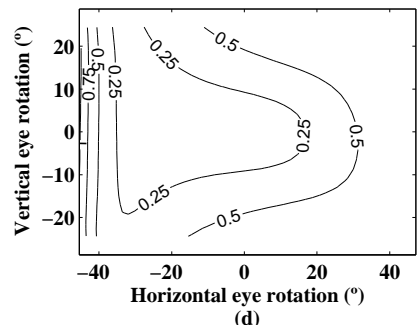

(d)

Figure 2. Power error as function of eye rotation for far (a) and near (c) vision. Cylinder error for far (b) and near (d) vision. +2 D Add design example.

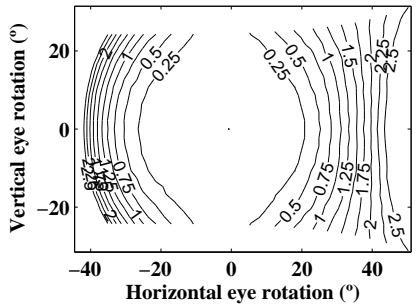

(a)

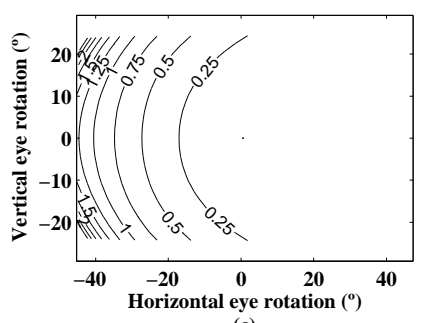

(c)

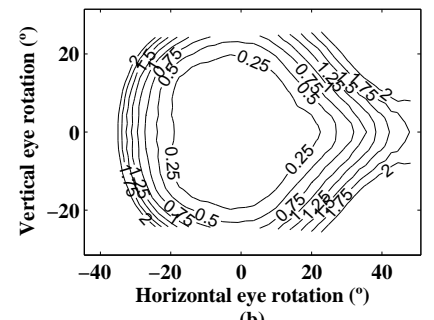

(b)

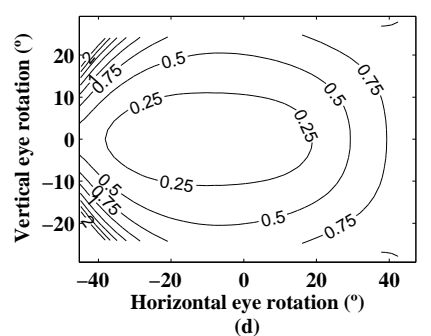

Figure 3. Power error as function of eye rotation for far (a) and near (c) vision. Cylinder error for far (b) and near (d) vision. $+3 \mathrm{D}$ Add design example.

For far vision both sphere and cylinder are still held less than 0.25 diopters within an elliptical optical window having major and minor axes of at least 40 degrees by 40 degrees of eye rotations. However for near vision the vertical dimension of of the optical window is slightly reduced to 30 degrees.

In our last design we reduced the addition to $+1.0 \mathrm{D}$. Accordingly the thicknesses are reduced: front lens $(1.4 \mathrm{~mm})$ and $2 \mathrm{~mm}$ the back lens. Fig. 3 shows the power (a-c) and cylinder (b-d) error distribution (deviations from nominal values) for far (a-b) and near (c-d) vision configuration.

The optical performance in now almost the same than in regular monofocal lenses. The error in sphere and cylinder is less than $0.25 \mathrm{D}$ within an elliptical optical window having major and minor axes of at least 45 degrees by 45 degrees of eye rotation. 


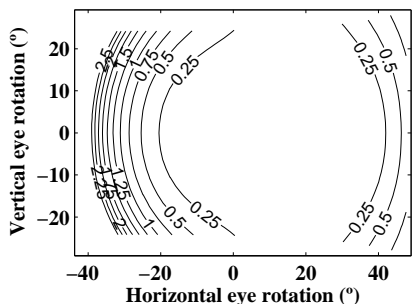

(a)

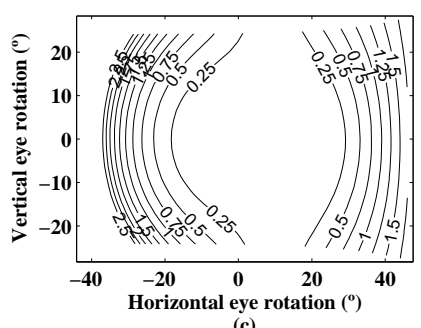

(c)

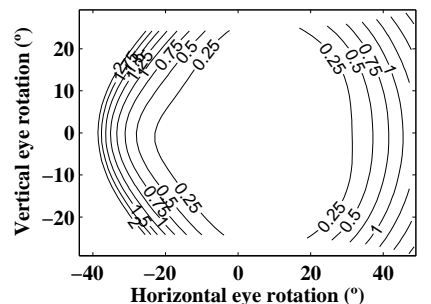

(b)

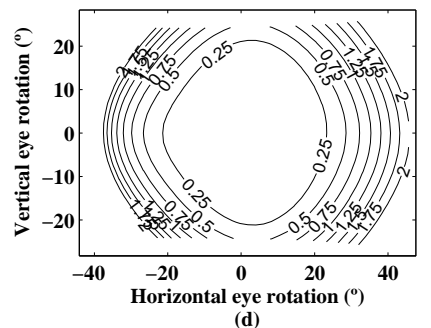

(d)

Figure 4. Power error as function of eye rotation for far (a) and near (c) vision. Cylinder error for far (b) and near (d) vision. +1 D Add design example.

\section{DISCUSSION}

We have presented a new way of designing bifocal wide field-of-view eyeglasses. For each object location the power is adjusted by moving transversely two lenses, one with respect to the other, in such a way that the typical field of-view available when looking through conventional monofocal eyeglasses is achieved. This technology represents a breakthrough over conventional bifocal lenses where the desired power is usable in only half part of the visual field.

In our design examples the achieved optical performance is slightly better for far vision with respect to near vision. The importance of precise cylinder is less crucial in near-distance vision tasks due to the depth of focus increase during accommodation. Therefore we consciously gave more weight to far vision in our merit function.

Although we do not provide any design example in this manuscript our methodology could be used to design trifocal field-of-view eyeglasses without loosing too much optical field-of-view.

\section{ACKNOWLEDGMENTS}

This work was supported by grants FIS2012-30820.

\section{APPENDIX A. INITIAL VALUES IN THE OPTIMIZATION}

For simplification let describe the non-planar anterior lens profile with only two terms:

$$
u(x, y)=A\left(\frac{x^{3}}{3}+x y^{2}\right)
$$

and similarly the non-planar posterior lens profile with:

$$
v(x, y)=A\left(\frac{x^{3}}{3}+x y^{2}\right)
$$

where $u$ and $v$ denote sag coordinate with respect to the vertex of the surface, so $u(x=0, y=0)=0$ and $v(x=0, y=0)=0$. The power at a surface hit point is given by: 


$$
P=2(n-1) A(x-\delta),
$$

where $\delta$ is the lateral shift of the lens surface.

Let $Q_{1}=\left(x_{1}, y_{1}, u\left(x_{1}, y_{1}\right)\right)$ and $Q_{2}=\left(x_{2}, y_{2}, v\left(x_{2}, y_{2}\right)\right)$ be the hit points of the wavefront with the front and back surfaces, respectively. Let $\delta_{u}$ and $\delta_{v}$ the lateral shifts of the anterior and the posterior lens respectively. Therefore the total power at a point $Q_{2}$ is under some approximations: ${ }^{4}$

$$
P\left(Q_{2}\right)=2(n-1) A\left(x_{1}-x_{2}+\delta_{v}-\delta_{u}\right),
$$

For a regular Alvarez design the gaze direction is always the same so the value of $x_{1}$ is fixed. However for a bifocal design $x_{1}$ changes for far and near vision. Typically if we set $x_{1}=0$ for far vision then $x_{1}^{\prime}=2.5$ for near vision.

Consider a configuration where $\delta_{u}$ and $\delta_{v}$ takes opposite values for far vision with respect to near vision. Then the power difference or Addition (Add) between both cases is given by:

$$
A d d=2(n-1) A\left(x_{1}-x_{1}^{\prime}+x_{2}^{\prime}-x_{2}+2 \delta_{u}-2 \delta_{v}\right),
$$

where $x_{1}^{\prime}, x_{2}^{\prime}$ are coordinates of the hit points when the eye look to near objects.

Using the thin lens approximation $\left(x_{1}=x_{2}\right.$ and $\left.x_{1}^{\prime}=x_{2}^{\prime}\right)$ the power is linearly dependent on $\delta_{u}-\delta_{v}$.

However the thin lens approximation is not accurate enough, ${ }^{4}$ so the goal is to find an approximation on the dependence of the power with the overall thickness $(\mathrm{t})$.

Assuming that $v\left(x_{2}, y_{2}\right)<<t$ and that the angle of incidence with respect to the normal to the surface is very small, the direction vector of the ray after refraction follows approximately the normal vector. Then $x_{2}-x_{1}$ can be estimated from $\frac{\partial u_{\delta}\left(x=x_{1}, y=0\right)}{\partial x}=A\left(x_{1}-\delta_{u}\right)^{2}$ using simple trigonometry:

$$
\begin{aligned}
& x_{2}-x_{1}=t A\left(x_{1}-\delta_{u}\right)^{2} \\
& x_{2}^{\prime}-x_{1}^{\prime}=t A\left(x_{1}^{\prime}+\delta_{u}\right)^{2}
\end{aligned}
$$

While this approximation is crude, it can be sued to obtain a better estimation than simply $x_{2}=x_{1}$ from the thin lens approximation. Substituting Eq. 8 and Eq. 9 in Eq. 7 we obtain:

$$
A d d=2(n-1) A\left(t A\left(x_{1}^{\prime 2}-x_{1}^{2}+2\left(x_{1}^{\prime}+x_{1}\right) \delta_{u}\right)+2 \delta_{u}-2 \delta_{v}\right),
$$

For the bifocal design, as described above, $x_{1}=0, x_{1}^{\prime}=e$, where $e$ denotes the eye convergence (mm). So Eq. 10 is transformed into:

$$
A d d=2(n-1) A\left(t A\left(e^{2}+2 e \delta_{u}\right)+2 \delta_{u}-2 \delta_{v}\right),
$$

Eq. 11 relates A with Add, t and $\delta_{u}$ and $\delta_{v}$.

If $t=0$ (thin lens) we get the usual equation:

$$
A=\frac{A d d}{4(n-1)\left(\delta_{u}-\delta_{v}\right)},
$$

If only the back lens is moved $\left(\delta_{u}=0\right)$ :

$$
A=\frac{2 \delta_{v}(n-1) \pm \sqrt{4 \delta_{v}^{2}(n-1)^{2}+2\left(e^{2}\right) \operatorname{Add}(n-1) t}}{2 e^{2}(n-1) t},
$$

In this equation there are two options to take either addition or the difference in the numerator. We select the difference. 


\section{REFERENCES}

[1] Jalie, M., [The principles of ophthalmic lenses], Association of Dispensing Opticians, London (1977).

[2] Weissman, B. and Morgan, M. W., "Marginal aberrations of different bifocal lens designs," Am. J. Optom. Physiol. Opt. 54(9), 588-594 (1977).

[3] Barnea, D., "Variable power lens and method," (USA Patent Number 4913536, 1990).

[4] Barbero, S. and Rubinstein, J., "Adjustable-focus lenses based on the alvarez principle," J. Opt. 13(12), 125705 (2011).

[5] Barbero, S. and Rubinstein, J., "Power-adjustable sphero-cylindrical refractor comprising two lenses," Opt. Eng. 52(6), 063002-063002 (2013).

[6] Eyejusters, "http://www.eyejusters.com."

[7] on Vision, F., "http://www.focus-on-vision.com."

[8] Adlens, "http://www.adlens.com."

[9] Peloux, M. and Berthelot, L., "Optimization of the optical performance of variable-power and astigmatism alvarez lenses," Appl. Opt. 53(29), 6670-6681 (2014).

[10] Zapata, A. and Barbero, S., "Mechanical design of a power-adjustable spectacle lens frame," J. Biomed. Opt. 16(5), 055001-6 (2011).

[11] Stone, B. D. and Forbes, G. W., "Characterization of 1st-order optical properties for asymmetric systems," J. Opt. Soc. Am. A 9(3), 478-489 (1992).

[12] Barbero, S., "Computation of dioptric and magnification matrices in ophthalmic lenses," J. Europ. Opt. Soc. Rap. Public. 9, 14023-5 (2014).

[13] Barbero, S. and Portilla, J., "Geometrical interpretation of dioptric blurring and magnification in ophthalmic lenses," Opt. Express 23(10), 13185-13199 (2015). 